\section{Revisión bibliográfica del aprovechamiento de lodos resultantes en el proceso de purificación de la sal: un acercamiento al comportamiento mecánico del lodo al interior de una mezcla de mortero de cemento hidráulico}

Bibliographic review of the use of resulting sludge in the salt purification process: an approach to the mechanical behavior of the sludge inside a mixture of hydraulic cement mortar

\section{Luis Eduardo Cock Bautista}

Corporación Universitaria Minuto de Dios -

Centro Regional Zipaquirá. Zipaquirá, Colombia

lcock@uniminuto.edu.co -

https://orcid.org/oooo-0003-2868-0848 -

\section{Gonzalo Andrés Flechas}

Corporación Universitaria Minuto de Dios -

Centro Regional Zipaquirá. Zipaquirá, Colombia gflechasgar@uniminuto.edu.co

Programa de Ingeniería Civil

\section{Resumen}

El artículo presenta una investigación exploratoria del tipo revisión bibliográfica sobre el estudio del aprovechamiento de residuos minerales del proceso de explotación de la sal gema como materia prima en ingeniería civil y un acercamiento al comportamiento mecánico de lodos de salmuera en una mezcla de mortero de cemento hidráulico. Tratar estos dos temas

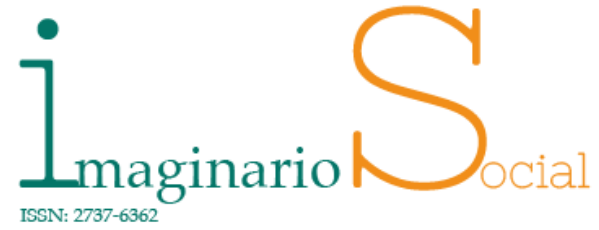

Imaginario Social

e-ISSN: $2737-6362$

julio - diciembre 2020 Vol. 3-2-2020 http://revista-

imaginariosocial.com/index.php/es/in dex

Recepción: 03 de abril 2019

Aceptación: 05 de junio 2020

1-16

Atribución/Reconocimiento-NoCo mercial- Compartirlgual 4.0 Licencia Pública Internacional - CC BY-NC-SA 4.0

https://creativecommons.org/licenses /by-nc- sa/4.0/legalcode.es 
tiene por objeto presentar posibles soluciones a la problemática ambiental que surge con la inadecuada disposición o uso final del residuo del proceso de fabricación de sal conocido como lodo de salmuera. Los resultados se obtienen en pruebas de resistencia a la compresión según norma NTC220 (Determinación de la resistencia de morteros de cemento hidráulico usando cubos de $50 \mathrm{~mm}$ o $50.8 \mathrm{~mm}$ de lado) con dos muestras para su análisis: una control y otra a la que se le adicionó lodo de salmuera resultante del proceso de purificación de la sal proveniente de las minas del municipio de Zipaquirá. Los resultados son alentadores en cuanto a que las dos muestras alcanzan aceptables resultados de resistencia a la edad de 28 días, además que la revisión bibliográfica arroja hallazgos relevantes para este comportamiento como una relación directa entre la concentración de cloruros en roca y la resistencia que presenta. En conclusión, este primer acercamiento del posible uso del material con fines ingenieriles invita a ahondar en sus usos, con sus correspondientes nuevos ensayos como es el caso de mejoramiento de bases en vías o su uso como material cementante suplementario.

Palabras clave: Lodos de salmuera, mortero, pruebas de resistencia, explotación, sal gema.

\section{Abstract}

The article presents an exploratory research of the bibliographic review type on the study of the use of mineral residues from the process of exploitation of gem salt as a raw material in civil engineering and an approach to the mechanical behavior of brine sludge in a mixture of hydraulic cement mortar. . Treating these two topics aims to present possible solutions to the environmental problem that arises with the improper disposal or final use of the residue from the salt manufacturing process known as brine mud. The results are obtained in tests of compressive strength according to the NTC220 standard (Determination of the resistance of hydraulic cement mortars using $50 \mathrm{~mm}$ or $50.8 \mathrm{~mm}$ cubes on each side) with two samples for analysis: one control and the other to which it added brine mud resulting from the purification process of the salt from the mines of the municipality of Zipaquirá. The results are encouraging in that the two samples achieve acceptable resistance results at the age of 28 days, in addition that the literature review yields relevant findings for this behavior as a direct relationship between the concentration of chloride in rock and the resistance it 
presents. .In conclusion, this first approach to the possible use of the material for engineering purposes invites us to delve into its uses, with its corresponding new tests, such as the improvement of road bases or its use as supplementary cementitious material.

Key word: Brine sludge, mortar, resistance tests, exploitation, rock salt.

\section{Introducción}

La sal es un producto esencial en la vida diaria y es tan común su uso que se puede encontrar en las cocinas de todos los hogares en diversidad de presentaciones. Además de ser un producto de consumo diario, también fue un elemento económico de gran impacto en épocas neolíticas o precolombinas (Filguns et al., 2013), ya que constituía un material con valor tanto social como económico en las regiones en las cuales se explotaba por lo que logró un rol de intercambio entre bienes y servicios en la época. Un claro ejemplo de ello fue el intercambio de sal de Zipaquirá por ollas de barro de Tocancipá: el historiador Andrés Olivos Lombana, en su libro Olleros y Sembradores, así lo cuenta: "Desde los tiempos muiscas y hasta finales del siglo XVII perduró el trueque. En este caso los indios de Tocancipá llevaban sus ollas y algo de leña, y a cambio recibían sal como pago".

Hoy en día la sal sigue teniendo protagonismo en la cotidianidad de las personas, es fundamental en la economía de la región de la sabana de Bogotá y está presente en la dieta de los hogares colombianos, en la manutención de animales y como materia prima de productos de aseo. Sirve en el control de la fermentación en procesos de productos lácteos; en la desinfección de aguas o en la industria química para la producción de cloro y soda cáustica, así como en la producción de sulfato de sodio y ácido clorhídrico (Agencia Nacional de Minería, 2019). En Colombia, como se observa en la siguiente tabla, tiene diferentes usos en la industria: 


\section{Tabla 1}

Usos industriales de la sal en Colombia. (Agencia Nacional de Minería, 2019)

\begin{tabular}{|llr|}
\hline Mineral & CIIU Sector Industrial & CIIU \\
\hline & Curtido y recurtido de cueros; recurtido y teñido de pieles & 1511 \\
& Elaboración de comidas y platos preparados & 1084 \\
& Elaboración de macarrones, fideos, alcuzcuz y productos & \\
& farináceos similares & 1083 \\
& Elaboración de otros productos alimenticios n.c.p. & 1089 \\
& Elaboración de productos de panadería & 1081 \\
& Fabricación de abonos y compuestos inorgánicos nitrogenados & 2012 \\
& Fabricación de jabones y detergentes, preparados para limpiar & \\
Sal 892 & y pulir; perfumes y preparados & 2023 \\
& Fabricación de otros productos químicos n.c.p. & 2029 \\
& Fabricación de sustancias y productos químicos básicos & 2011 \\
& Preparación e hilatura de fibras textiles & 1311 \\
& Procesamiento y conservación de carne y productos cárnicos & 1011 \\
& Procesamiento y conservación de frutas, legumbres, hortalizas & \\
& y tubérculos & 1020 \\
& Procesamiento y conservación de pescados, crustáceos y y & 1012 \\
& moluscos & \\
\hline
\end{tabular}

La sal, denominada químicamente como Cloruro de sodio, bajo la fórmula $\mathrm{NaCl}$, es un mineral que se encuentra en la naturaleza y sus reservas son consideradas incalculables dada su variedad de fuentes de obtención (Agencia Nacional de Minería, 2019) como los océanos en donde la sal marina se extrae por secado, a través de evaporación solar, o mediante la extracción minera de sal gema, también conocida como halita, mediante procesos más avanzados como la disolución in-situ de la cual se hablará más adelante.

\subsection{Explotación de la sal a nivel mundial y local}

Una de las explotaciones más antiguas de sal se encuentra en Muntanya de Sal en el municipio de Cardona, perteneciente a la provincia de Barcelona en España. Se trata de la primera mina de Europa Occidental que inició actividad entre 4200 a 4500 a. C. (Filguns et al., 2013) y cuyo método de explotación fue a través del medio mecánico usando piedras pulidas como herramientas de tallado. En la actualidad los países que lideran la producción salina son China y Estados Unidos con una producción anual de 69 y 43 millones de toneladas respectivamente, lo que corresponde al $40 \%$ de la explotación mundial (Agencia Nacional de Minería, 2019), les sigue India con 20 millones de toneladas y junto a éstos Canadá, Alemania, Australia y Chile.

Colombia tiene una producción baja en comparación con estos países ya que su explotación salinera anual está en un promedio de 1,48 millones de toneladas, lo que corresponde a 0,2 \% de la producción mundial (Agencia Nacional de Minería, 2019). 
Las cinco concesiones que se observan en la siguiente tabla son las encargadas de dicha extracción minera:

Tabla 2

Concesiones de sal en Colombia. Valora consultoría S.A.S

\begin{tabular}{|lll|}
\hline Contrato & Nombre concesión & Ubicación \\
\hline DID-082 & Brinsa S.A. & Sesquilé \\
HIQO-03 & Colsalminas Ltda & Zipaquirá \\
HIQO-01 & Colsalminas Ltda & Nemocón \\
HINM-01 & Sama Ltda & Manaure \\
UPIN-HIQL-01 & Salcol S.A. & Restrepo* \\
\hline
\end{tabular}

De estas concesiones, cuatro se refieren a explotación de sal gema y solo una (Manaure) corresponde a producción de sal marina. Según indicadores de la Agencia Nacional de Minería (2019) la demanda de sal en Colombia corresponde a 2.154.225 toneladas para el año 2016. Según su origen, el 95\% de dicha producción es de sal marina sin purificar y el $5 \%$ restante representa la extracción de sal gema o de mina; no obstante a tan marcada diferencia, la demanda de esta última es significativa por su uso como producto básico de la canasta familiar, luego de un proceso de refinación. El mercado de la extracción de sal en la región de la Sabana de Bogotá, está dirigido principalmente al refinamiento para consumo humano y como complemento alimenticio en animales, principalmente ganado vacuno y equino. La concesión Brinsa S.A.S (Agencia Nacional de Minería, 2019) abarca el 87 \% del mercado, con una preferencia del 92\% de los consumidores. El mecanismo de obtención de la sal en esta concesión es a partir de la disolución in-situ que proporciona un producto más puro y con mejor relación de costo eficiencia en la producción de sal para el consumo humano. La Agencia Nacional de Minería (2019) reporta un aumento de demanda de sal de consumo humano en Colombia, en los últimos cuatro años. El hecho de que la sal haga parte de la canasta familiar en Colombia y que además mantenga su crecimiento de demanda asegura un mercado estable y con proyección de extracción minera por muchos años más.

Es innegable que la sal continúa siendo un importante sector de la economía colombiana y que en respuesta a su demanda la extracción de sal de gema se seguirá 
haciendo por lo que optimizar el manejo de los residuos de su producción se convierte en un reto para esta industria.

\subsection{Descripción geológica del domo y explotación de la sal en Zipaquirá. Concesión Colsaminas Ltda}

Según Amaya y Pérez (2016), las salinas de Zipaquirá corresponden a un domo originado en la formación geológica Chipaque donde el suelo se caracteriza por presentar limolitas negras y arcillolitas negras bituminosas que son ricas en cristales de piritas y algunas capas de lulitas que en lenguaje minero se conocen como rute y hacen parte del estéril del yacimiento. La halita corresponde a un material característico por sus cristales de color blanco, gris claro y oscuro que varía dependiendo de la cantidad de impurezas carbonosas-arcillosas y que presenta una composición de 88 \% de cloruro de sodio. Cabe resaltar que la composición del domo se caracteriza en gran parte por el contenido de fragmentos de arcillolita que corresponden a un material arcilloso asociado con el depósito de sal que fue formado por el efecto de la erosión y que se presenta en la floculación de la salmuera en el proceso de purificación de la sal.

Las excavaciones están divididas en cuatro niveles que corresponden a Guasá, Potosí, Frabricalta y Subsabana, los dos últimos niveles actualmente se encuentran en explotación. En el nivel Subsabana hay cinco pozos de explotación por disolución Insitu, de los cuales dos están activos (Amaya y Pérez, 2016) y de los cuales se obtiene la salmuera para su posterior purificación en tanques de almacenamiento.

En los dos pozos del nivel Subsabana se extrae sal con un 15 \% de materia orgánica en su estado natural que es necesario purificar para el consumo humano y animal. Esto se hace a través del proceso de disolución In-Situ, técnica favorable económicamente pues evita la explotación mecánica que es más costosa por la necesidad de excavaciones (Amaya y Pérez, 2106) y consiste en la inyección de agua dulce por un tubo de 3" para su disolución de cloruros de sodio, el agua salada denominada salmuera sube por la diferencia de presiones a través de la tubería de 6" denominada tubería de producción, el líquido aislante se bombea durante el proceso con el fin de 
crear una capa aislante y resistente para evitar el colapso del pozo como se observa en la figura 1. (Colsalminas, 2018).

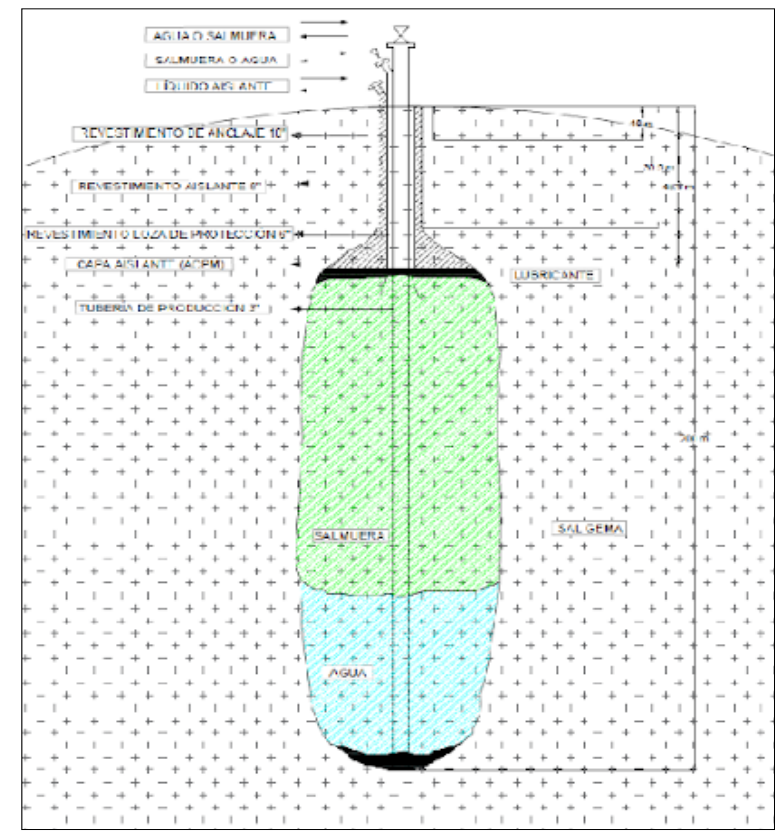

Figura 1. Sistema de explotación por disolución in-situ. Colsalminas Ldta La sal gema es disuelta en agua fría o caliente para separarla del material rute. La salmuera cruda resultante de este proceso se transporta a tanques o piscinas de almacenamiento donde por procesos fisicoquímicos se purifica al añadir carbonato de sodio y soda cáustica que precipitan la materia orgánica y los trazos de oligoelementos. Finalmente, se pasa a una etapa de evaporación en donde la acción de grandes temperaturas producidas por una caldera elimina el agua para obtener granos de sal blancos y finos. (Colsalminas, 2018)

\subsection{Residuos de la explotación sal gema de Zipaquirá y su impacto en el ambiente}

La actividad minera causa impactos ambientales negativos por la generación de residuos en sus procesos de explotación. En la actualidad son limitadas las 
investigaciones que se centran en el estudio del comportamiento de dichos residuos, en especial en aquellos que se generan en la explotación de la sal gema.

De acuerdo con Rodas (2007) en España la explotación de la sal ha provocado grandes impactos por la generación de desechos mineros que se encuentran dispuestos en escombreras que pueden alcanzar los 100 metros de altura. El autor aclara que la mayoría de estos residuos tienen comportamientos diferentes a los de un suelo ya que, entre otros aspectos, tienen un peso específico menor, una alta solubilidad en el agua y una capacidad importante de cementación. Conocer las propiedades geotécnicas de estos materiales es fundamental para determinar la estabilidad de los centros de acopio de los residuos mineros de la sal que teniendo presente que se clasifican como lodos residuales.

Hernández et al. (2006) manifiestan que lodos son residuos de difícil disposición por sus componentes tanto físicos como químicos y la falta de lugares de acopio hace de estos un material de tratamiento especial. Colombia no es la excepción considerando que es un país donde la extracción minera de la sal es activa. Actualmente, en la producción de sal en las minas de Zipaquirá, se genera 800 kilogramos de lodos al día (Colsalminas, 2019) los cuales requieren de una disposición final adecuada para mitigar los efectos en el medio ambiente, puntualmente en el suelo ya que, al contener una alta concentración de cloruro de sodio, hace de este residuo minero un componente que afecta la fotosíntesis de la capa vegetal y de las plantas causando deterioro y desertificación del terreno al cambiar las características físicas y químicas del suelo (Olivia et al., 2008), además de la posible filtración de los elementos minerales a los cuerpos hídricos en la zona afectando su composición y posible potabilidad (Malcebo, 2008).

Para el caso de las minas de sal de Zipaquirá, la generación de los lodos se presenta entre el proceso de purificación de la sal gema en donde a través de la aplicación de la soda cáustica y carbonato de sodio se obtiene un residuo lodoso cuya disposición, manejo y tratamiento son del todo ausentes al no contar con escombreras especializadas para el acopio de este material subproducto del proceso de la sal yodada. (Colsalminas, 2018) 
1.5 Aprovechamiento de los residuos minerales en la mina de sal de Zipaquirá

En la actualidad no se presenta ningún proyecto o propuesta dirigida al aprovechamiento de los residuos minerales generados por la explotación de sal en Colombia y más precisamente en municipios como Zipaquirá, Sesquilé y Nemocón. Se encuentra un campo de estudio no explorado y abierto a posibles investigaciones del comportamiento mecánico de los lodos provenientes de las minas de sal de gema de esta región. En el documento "Obtención del yeso presente en las piscinas de salmuera, su posterior extracción y comercialización para la empresa ecuatoriana de sal" (Rodriguez, 2016) se demuestra la importancia de consolidar un estudio de los residuos salinos y de esta manera realizar su aprovechamiento en aspectos de ingeniería civil como lo manifiesta Rodríguez (2016) en donde el yeso obtenido de la decantación de la sal marina es utilizado como material adicional en la producción del cemento o en la construcción de paneles de yeso para paredes.

Por otro lado el uso del Cloruro de Sodio, como estabilizante en la estructura del pavimento, en la construcción de vías es un componente que aumenta el tiempo de humedad para aquellos suelos que lo requieren, ya que es capaz de absorber la humedad del ambiente creando una barrera para evitar que se evapore rápidamente $\mathrm{e}$ incrementando la densidad seca máxima a la vez que reduce la humedad óptima. (Roldán, 2010). Esta caracterización del cloruro de sodio en actividad de la ingeniería civil, propicia una pregunta de investigación en relación a la pertinencia del posible uso de lodos en la construcción de bases y sub-bases por su alto contenido de cloruro de sodio.

Teniendo en consideración lo expuesto y dada la poca bibliografía relacionada con el tema de discusión, es pertinente realizar una exploración del comportamiento mecánico del material residuo mineral de la sal gema como adición a de una mezcla de mortero y realizar pruebas de laboratorio de acuerdo con la normativa NTC 220 con la variación de adicionar $25 \%$ del lodo seco en relación a la cantidad de cemento UG sugerido por la norma, con el fin de identificar la variación de la resistencia a las edades de 7, 14 y 28 días. 


\section{Materiales y Métodos}

\subsection{Caracterización del material}

La empresa Colsalminas ltda realizó una caracterización del lodo de salmuera en el año 2012 a través de un laboratorio acreditado con los estándares técnicos de calidad, dicha caracterización muestra una variedad de sustancias que componen el residuo extraído del tanque de tratamiento de salmuera de la mina de sal en el municipio de Zipaquirá. En la tabla 3, correspondiente a la caracterización de este material, se evidencia un alto contenido de cloruros. Tabla 3

Caracterización de residuo lodo de salmuera. Colsalminas (2018)

\begin{tabular}{|c|c|c|c|}
\hline PARAMETRO & UNIDADES & RESULTADO & METODO ANALITICO \\
\hline ALUMINIO TCLP & $\mathrm{mg} / \mathrm{Kg}$ de $\mathrm{Al} 3+$ & $<2.00$ & SM 3111 D \\
\hline CADMIO TCLP & $\mathrm{mg} / \mathrm{L} \mathrm{de} \mathrm{Cd}$ & $<0.04$ & SM 3111 B \\
\hline CLORUROS & $\mathrm{mg} / \mathrm{Kg}$ de Cl- & 5876.4 & SM $4500 \mathrm{Cl}-\mathrm{B}$ \\
\hline COBRE TCLP & $\mathrm{mg} / \mathrm{L}$ de $\mathrm{Cu}$ & $<0.10$ & SM 3111 B \\
\hline CROMO TCLP & $\mathrm{mg} / \mathrm{L}$ de $\mathrm{Cr}$ & $<0.20$ & SM 3111 B \\
\hline CROMO VI (SUB) & $\mathrm{mg} / \mathrm{L}$ de $\mathrm{Cr} \mathrm{VI}$ & $<0.02$ & COLORIMETRÍA \\
\hline HIERRO TCLP & $\mathrm{mg} / \mathrm{Kg}$ de $\mathrm{Fe}$ & $<0.20$ & SM 3111 B \\
\hline MERCURIO TCLP (SUB) & $\mathrm{mg} / \mathrm{L}$ de $\mathrm{Hg}$ & 0.000488 & SM $3114 \mathrm{~B}$ \\
\hline NIQUEL TCLP & $\mathrm{mg} / \mathrm{L}$ de $\mathrm{Ni}$ & $<0.30$ & SM 3111 B \\
\hline $\mathrm{PH}$ & Unidades de $\mathrm{pH}$ & 12.43 & SM $4500 \mathrm{H}-\mathrm{B}$ \\
\hline PLOMO TCLP & $\mathrm{mg} / \mathrm{L}$ de $\mathrm{Pb}$ & 0.44 & SM 3111 B \\
\hline ZINC TCLP & $\mathrm{mg} / \mathrm{L}$ de $\mathrm{Zn}$ & 1.07 & SM 3111 B \\
\hline
\end{tabular}

\subsection{Secado y molienda}

Las pruebas se realizaron en el laboratorio de suelos de la Corporación Universitaria Minuto de Dios, ubicada en el municipio de Zipaquirá, Colombia; a una altura de 2650 metros sobre el nivel del mar y una temperatura promedio de $14^{\circ} \mathrm{C}$.

El lodo de salmuera proveniente de la mina de sal de Zipaquirá, bajo el consorcio de explotación Colsalminas, se llevó a un horno eléctrico a una temperatura de 105 grados Celsius durante 24 horas (figura 2) con la finalidad de obtener un material seco, posteriormente se pasó el material por un tamiz de 300 micras (figura 3). El material mayor al diámetro de la malla se trituró con un martillo de goma hasta obtener la finura homogénea adecuada para la mezcla. 


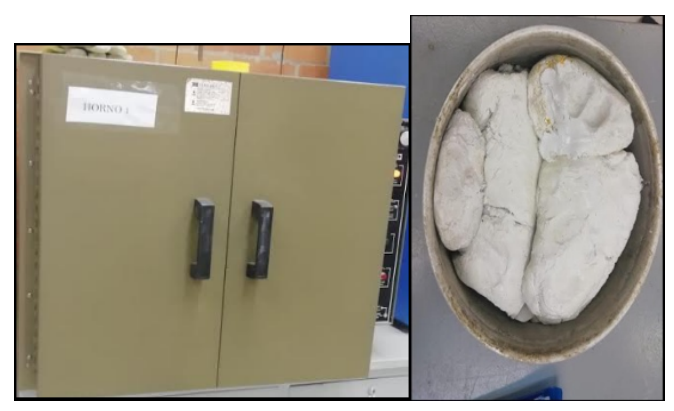

Figura 2. Proceso de secado del lodo. Laboratorio de Suelos, Corporación Universitaria Minuto de Dios, Sede Zipaquirá.

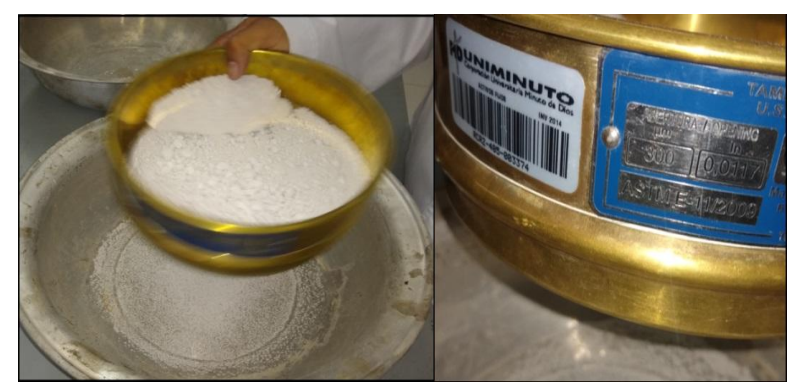

Figura 3. Proceso de tamizado del material. Laboratorio de Materiales Corporación Universitaria Minuto de Dios, sede Zipaquirá.

\subsection{Mezcla y especímenes}

Para comparar el comportamiento mecánico del mortero con y sin adición de lodo de salmuera se procedió a realizar una prueba de acuerdo con la norma NTC 220. Se formaron dos especímenes: el tipo 1 al que se le adicionó un 25\% de lodo de salmuera y el tipo 2 es la muestra control de acuerdo con la mezcla típica sugerida en la norma, respecto a la cantidad de cemento dispuesta en la norma.

Para el espécimen tipo 1, se formaron nueve cubos de mortero, se le adicionó un 25\% de lodo de salmuera respecto a la cantidad sugerida en la norma para cemento; y se dejó la composición sugerida en la norma para el resto de materiales, tal como se observa en la siguiente tabla: 
Tabla 4 Composición de los morteros con adición de Lodo de Salmuera, espécimen 1.

\begin{tabular}{|l|l|}
\hline Material & $\begin{array}{l}9 \\
\text { cubos }\end{array}$ \\
\hline Cemento UG, g & 740 \\
\hline Arena, g & 2035 \\
\hline Agua si se usa cemento UG, ml. & 242 \\
\hline Lodo de Salmuera, g. & 185 \\
\hline
\end{tabular}

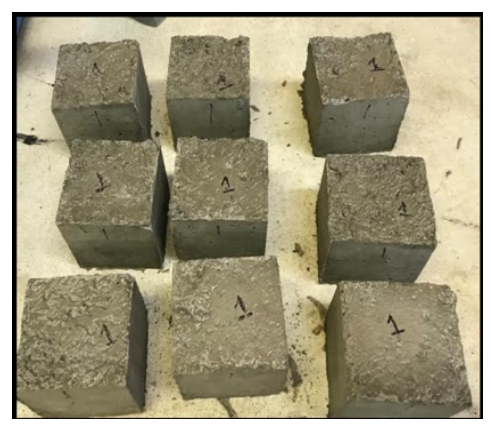

Figura 4. Espécimen Tipo 1

Para el caso del espécimen tipo 2 muestra control, igualmente se formaron nueve cubos de mortero con la siguiente composición de acuerdo con la norma NTC220 (tabla 5):

Tabla 5 Composición de los morteros, espécimen 2. Norma Técnica Colombiana, NTC 220.

\begin{tabular}{|l|l|}
\hline Material & $\begin{array}{l}9 \\
\text { cubos }\end{array}$ \\
\hline Cemento UG, g & 740 \\
\hline Arena, g & 2035 \\
\hline Agua si se usa cemento UG, ml. & 242 \\
\hline
\end{tabular}




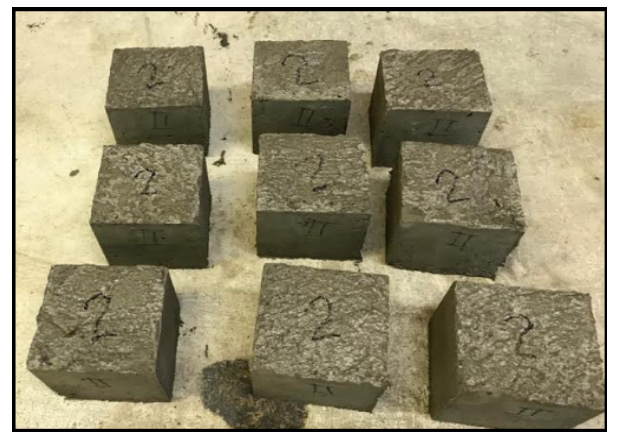

Figura 5. Espécimen Tipo 2.

El Instituto Colombiano de Normas Técnicas y Certificación ICONTEC, NTC 220, resume este método de ensayo de la siguiente forma: "Las proporciones en masa para formar el mortero usado están compuestas de una parte de cemento y 2,75 partes de arena. Los cementos Pórtland con incorporadores de aire son mezclados con una relación agua/cemento especificada. Los cubos de ensayo de $50 \mathrm{~mm}$ ó 50,8 $\mathrm{mm}$ se apisonan en dos capas. Los cubos son curados un día en los moldes, luego se desmoldan y se sumergen en agua con cal hasta el momento del ensayo.”

Cada uno de los dos grupos de 9 cubos fue enviado a un laboratorio acreditado para ser fallados a 7, 14 y 28 días de edad para conocer su carga y resistencia.

\section{Resultados}

Las pruebas de compresión, tanto para el espécimen control como para el que tuvo una adición de lodo de salmuera, realizadas para edades de 7, 14 y 28 días, tiene tendencias de crecimiento, y en los dos casos alcanzaron más de 28 MPA de resistencia a los 28 días de edad.

Denota especial comportamiento el espécimen 1 (figura 6), que contiene la adición de lodo de salmuera ya que en la edad de 7 y 14 días alcanzó un mejor comportamiento que la muestra control respecto a la resistencia alcanzada en $17 \%$ y $14 \%$ respectivamente; mientras que a los 28 días de edad cambió la tendencia encontrándose un mejor comportamiento de la muestra control en un $5 \%$. 


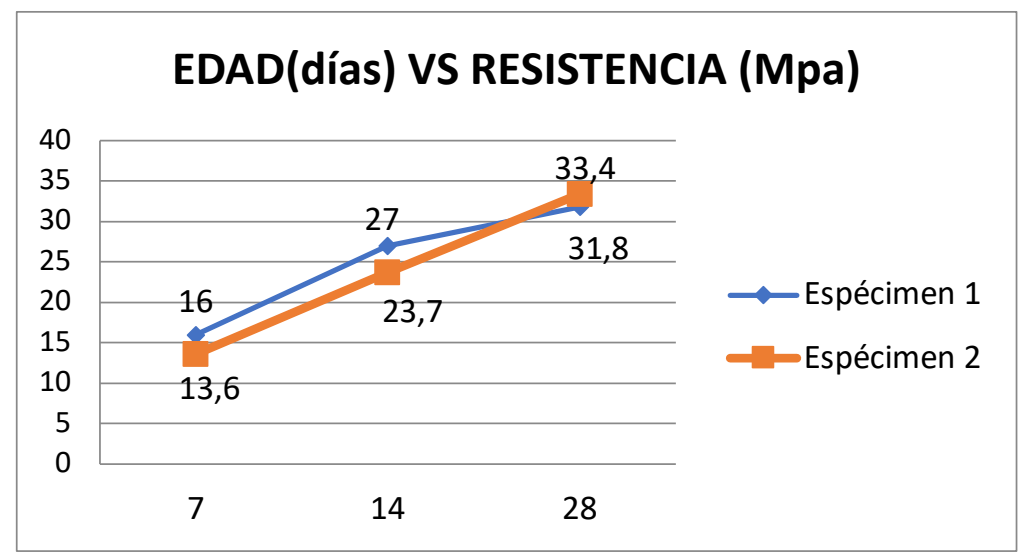

Figura 6: Resultados Edad Vs. Resistencia.

Ahora bien, el similar comportamiento de las muestras describe un funcionamiento aceptable del nuevo material adicionado. Con estos resultados se torna evidente realizar el ensayo cambiando parte de la dosificación de cemento con el material objeto de estudio para darle uso como un tipo de MCS Material Cementante Suplementario.

Sin embargo, no es del todo claro el comportamiento del espécimen tipo 2 después de los 14 días ya que se observa un cambio leve en la tendencia de muestra su curva de resistencia. Se requiere optar por nuevos estudios, así como fallar los cubos a mayor edad.

Es evidente, dada la presente revisión bibliográfica, que el aprovechamiento de los residuos provenientes del proceso de extracción de la sal de gema, puntualmente del lodo de salmuera, es benéfico para el medio ambiente, toda vez que no hay un lugar de disposición final de dicho material. Los alentadores resultados de su uso como material cementante suplementario invitan a ahondar en su estudio.

\section{Discusión}

La presente revisión bibliográfica muestra que no se cuenta con suficientes estudios publicados sobre el aprovechamiento del lodo de salmuera proveniente del proceso de fabricación de sal por el método in-situ en minas de sal de gema. Teniendo en cuenta el impacto negativo que este material presenta, principalmente en los suelos donde se hace su disposición final, es recomendable ahondar en esta y otras posibles investigaciones que tengan por objeto el aprovechamiento de este material en otros procesos productivos. 
Los resultados del ensayo basado en la norma NTC 220 fueron aceptables para la muestra con proporción del 25\% de lodo de salmuera adicionado respecto a la cantidad de cemento que determina esta norma por tener comportamientos similares con la muestra control. Dichos resultados motivan a continuar haciendo estudios sobre el uso de este material en el mortero hidráulico. Para su uso como MCS se requiere que los nuevos estudios no tengan la dosificación de lodo de sal como adición a la mezcla sino como material suplementario en ella.

Según Colsalminas (2018) el comportamiento mecánico de la roca en referencia a la resistencia está determinado por el contenido de cloruro, es decir a mayor concentración de cloruro de sodio mayor será también su resistencia lo que podría argumentar el comportamiento particular de los lodos de salmuera en las dos primeras edades, sin embargo es necesario observar si dicha dinámica es constante con porcentajes de contenido de lodo a 50\% o $75 \%$ en la mezcla de mortero.

\section{Referencias}

Alvarez, C. (2018). Caracterización de una mezcla de mortero a base de Azufre no modificado. Universidad del Norte.

Amaya, R., \& Perez, D. (2016). Elaboración delmodelo hidrogeológico conceptual de la mina de sal de Zipaquirá. UPTC.

Camacho, D., \& Arias, V. (2016). Evaluación de desempeño de una mezcla de concreto muestra patrón, muestra mezcla con sal al 3,5 \% y muestra sumergida en agua con sal al 3,5\%. Universidad Católica.

Colsalminas. (2018). Plan de Manejo Ambiental. Colsalminas.

Filguns, A., Weller, O., Grandia, F., Bonache, J., \& Gonzalez, J. (2013). La primera explotación de sal gema: la Vall salina de Cardona. Revista antropología chilena , 177-195.

Hernandez, D., Villegas, J., Castaño, J., \& Paredes, D. (2006). Aprovechamiento de losdos aluminosos generados en sistemas de potabilización, mediante su corporacón como agregado en materiales de construcción. Revista Ingeneirías. Universidad de Medellín, 119-132. 
Malcebo, J. (2008). Karstología de yesos. Aplicaciones a la ingeniería civíl. Universidad Politécnica de Madrid.

Minería, A. d. (2019). Estudios previso. Minenergía.

Oliva, M., Rincón, R., Zenteno, E., \& Pinto, A. (2008). Rol del vermicompostfrente al estres por cloruro de sodio en el crecimiento y la fotosíntesis en plantulas de tamarindo. Gayana BOT, 10-17.

Rincón, C., Gil, J., Lesmes, C., Caro, \& Carlos. (2016). Evaluación de la sostenibilidad de la producción de ladrilloen la región de Boyacá, Colombia. L'esprit Ingeniux, 35-45.

Rodas, E. (2007). Caracterización geotécnica de los residuos de minas de sal. Departament Enginyeria del Terreny, 12-58.

Rodriguez, J. (2016). Obtención del yeso presente en las piscinas de sal muera, su posterior extracción y comercialización para la empresa Ecuatoriana de Sal y Productos Químicos en la Península de Santa Elena. Universidad Espíritu Santo.

Roldán, J. (2010). Estabilización de suelos con cloruro de sodio para bases y subbases. Universidad de San Carlos Guatemala.

Rubio, R. (2006). El material de yeso: comportamiento y conservación. Cuadernos de restauración, 60-62. 